ORIGINAL ARTICLE

\title{
Results of the guideline-based treatment for pemphigus: a single-centre experience with 84 cases
}

\author{
R. Kakuta, (ID Y. Kurihara, J. Yamagami, ${ }^{\star}$ J. Miyamoto, T. Funakoshi, A. Tanikawa, M. Amagai \\ Department of Dermatology, Keio University School of Medicine, Tokyo, Japan \\ *Correspondence: J. Yamagami. E-mail: yamagamijun@keio.jp
}

\begin{abstract}
Background The Japanese guidelines for the management of pemphigus (JG) were published in 2010. However, further progress in the treatment of pemphigus requires their validation.

Objectives To examine the efficacy and safety of treatments based on the JG.

Methods A retrospective study of 84 Japanese patients with moderate to severe pemphigus, who were initially treated in accordance with the JG and then followed up for $>2$ years, was performed in a single centre. Treatment typically consisted of $0.5-1 \mathrm{mg}$ prednisone (PSL)/kg/day accompanied by $100 \mathrm{mg}$ azathioprine/day as a steroid-sparing agent.

Results In 83 of the 84 patients (98.8\%), complete remission on minimal therapy ( $\leq 10 \mathrm{mg}$ PSL/day and concomitant immunosuppressive agent) was achieved. The time between initiation of therapy and remission was $13.9 \pm 9.4$ months. In 78 patients (92.9\%), remission was accomplished within the 2-year follow-up. The 32 patients with recalcitrant disease (38.1\%) received additional treatment. Relapse occurred in 12 patients (14.3\%) either during tapering of the PSL dose (six patients) or after achieving remission (six patients). Adverse events, mostly liver enzyme elevation, infections and diabetes, occurred in 67 patients (79.8\%). One patient (1.2\%) died during the observation period after gastrointestinal haemorrhage.
\end{abstract}

Conclusions Our results suggested that the elderly and patients requiring additional therapies were at higher risk of adverse events, including severe infections, and should thus be monitored carefully. This study provided clinical data that could inform revised guidelines and contribute to the evaluation of future novel therapies.

Received: 17 July 2019; Accepted: 16 December 2019

\section{Conflicts of interest}

No conflicts of interest to declare.

\section{Funding sources}

Health and Labor Sciences Research Grants for Research on Measures for Intractable Disease from the Ministry of Health, Labor and Welfare of Japan, and Intramural research fund of Keio University School of Medicine.

\section{Introduction}

Pemphigus is an autoimmune disease characterized by the formation of blisters induced by anti-desmoglein (Dsg) autoantibodies. Previously, pemphigus was fatal, but the introduction of systemic corticosteroids (CS) as therapy has markedly reduced the mortality rate, and the prognosis has improved in most patients. ${ }^{1}$ However, high doses and prolonged use of CS can result in adverse events. To minimize these risks, adjuvant therapies, including immunosuppressive agents, high-dose intravenous immunoglobulin (IVIG) and plasma exchange (PE), have been added to therapeutic regimens, allowing reduction of daily and total CS doses.

Given the rarity of pemphigus, strategies for its treatment have been lacking for many years. In the absence of reliable measurement tools and flexible treatment plans, including with regard to CS dosage and use of drugs in combination, expert experience has been relied on to estimate disease activities based on the clinical appearance of affected patients. To improve and standardize the care of pemphigus patients on a worldwide scale, guidelines for the management and treatment of pemphigus have been sought. The British Association of Dermatologists was the first group to publish guidelines for the management of pemphigus vulgaris (PV), ${ }^{2}$ followed by the publication of six additional, updated guidelines. ${ }^{3-9}$ Furthermore, the International Pemphigus Committee ${ }^{10}$ released a consensus statement that has been applied to define both the common terms used to evaluate pemphigus and the treatment endpoints of the disease. The information contained in the statement has allowed experts in the field to accurately measure and assess disease severity, as well as therapeutic responses. Nonetheless, because of the rarity 
of the disease, well-designed studies contributing to evidencebased practice have been lacking. Further evolution of the treatment strategy for pemphigus requires assessment and validation of the current guidelines.

Pemphigus patients seen in our department are treated strictly according to the Japanese guidelines for the management of pemphigus (JG), ${ }^{4}$ which state that the goal of treatment is remission on therapy equivalent to a prednisone (PSL) dose of $\leq 10 \mathrm{mg} /$ day and minimal adjuvant therapy. To achieve this goal, an initial CS dose of $1 \mathrm{mg}$ PSL $/ \mathrm{kg} /$ day is recommended for patients with moderate to severe disease activity. Treatment should be divided into two stages: a consolidation phase (initial therapy), in which treatment is adjusted until the disease is controlled, including initial tapering of the CS dose; and a maintenance phase (maintenance therapy), during which treatment is maintained and tapering of the CS dose is well-established. Additional therapy (e.g. PE, IVIG) should be provided, without an increase in the daily CS dose, if initial therapy is not sufficient to control disease activity. Disease activity can be monitored objectively using the Pemphigus Disease Area Index (PDAI), given its high convergent validity, reproducibility and correlations with the treating physician's impression of disease activity. ${ }^{11,12}$

In this study, we retrospectively investigated the efficacy, safety and outcomes of therapies administered in accordance with the JG. Our goal was to determine whether pemphigus patients benefit from JG-based treatment.

\section{Methods}

\section{Patients}

We retrospectively assessed 84 Japanese patients with pemphigus treated based on the JG at the Department of Dermatology in Keio University between 2009 and 2015. The study was approved by the Institutional Review Board of Keio University and was conducted according to all relevant requirements of the Declaration of Helsinki. All patients satisfied the following criteria: diagnosis of pemphigus confirmed clinically (presence of blisters and erosions on the skin and mucous membranes), histopathologically (intraepidermal blister formation with acantholysis) and immunologically (intercellular IgG autoantibody deposition as revealed by direct and/or indirect immunofluorescence); moderate to severe disease activity; and $>2$ years of follow-up after the initiation of therapy. All patients had the typical clinical and histopathological characteristics of pemphigus.

\section{Evaluation of disease activity and serum autoantibodies}

During the observation period, clinical disease activity was evaluated using the PDAI, a scoring system that indexes the extent and severity of mucous and cutaneous lesions. ${ }^{12}$ Severity grade before initial treatment was determined according to the PDAI (0-8, mild; 9-24, moderate; and $\geq 25$, severe activity ${ }^{13}$ ). Serum autoantibody levels were measured quantitatively by enzyme-linked immunosorbent assays (ELISAs), with ELISA index obtained for autoantibodies against Dsg1 or Dsg3 in PV and pemphigus vegetans, and against Dsg1 in pemphigus foliaceus $(\mathrm{PF})$.

\section{Treatment}

In accordance with the recommendations of the JG, the initial daily dose of PSL was 1 or $0.5 \mathrm{mg} / \mathrm{kg} /$ day, and that of the immunosuppressive agent azathioprine (AZP) was $100 \mathrm{mg} / \mathrm{day}^{4}$ Topical CS (group III) and antibiotics were also applied concomitantly to cutaneous and mucous lesions in some patients. If the initial treatment was determined to be insufficient after 2 weeks, additional treatment consisting of PE, steroid pulse therapy and/or IVIG was administered. If the initial therapy succeeded in controlling disease activity and inducing the end of the consolidation phase, the oral CS dose was tapered as follows: reduction of 5-10 mg/1-2 weeks if > $20 \mathrm{mg}$ PSL/day had been prescribed, and of 1-3 mg/1-2 months if the prescribed dose was $\leq 20 \mathrm{mg}$ PSL/day. After the goal PSL dose (10 mg/day) had been reached, immunosuppressive agents were gradually reduced followed by tapering of the PSL dose to the maintenance dose at a rate of $<5 \mathrm{mg} /$ day. For patients with persistent blister formation despite repeated treatment, the administration of human anti-CD20 monoclonal antibody was considered.

\section{Definitions and response to treatment}

Remission was defined as the absence of new and/or established lesions for at least 2 months in patients receiving $\leq 10 \mathrm{mg}$ PSL/day and concomitant use of minimal adjuvant therapy [complete remission $(\mathrm{CR})$ on minimal therapy ${ }^{10}$ ]. The number of cases of remission was counted to verify the effectiveness of the JG. Disease relapse was defined as the development of $\geq 3$ new lesions per month with no spontaneous healing within 1 week, or the extension and enlargement of existing lesions in a patient in whom disease activity had previously been controlled.

\section{Statistical analyses}

The results are expressed as the mean \pm standard deviation (SD). The association between intervention and outcome in the groups that did and did not require additional therapy was evaluated using Fisher's exact test. The appropriate parameters were compared using Welch's $t$-test. The distribution of CR in response to therapy was analysed using the Kaplan-Meier method, and the log-rank test was used to compare the two groups that did and did not require additional therapy. In all analyses, $P<0.05$ was taken to indicate statistical significance.

\section{Results}

\section{Patient characteristics}

The 84 patients enrolled in this study included 53 with PV, 28 with PF and 3 with pemphigus vegetans (Table 1). The follow-up 
Table 1 Patient characteristics in this study

\begin{tabular}{|c|c|c|c|c|}
\hline & All cases $(n=84)$ & $\begin{array}{l}\text { Additional therapy }(-) \\
(n=52)\end{array}$ & $\begin{array}{l}\text { Additional therapy }(+) \\
(n=32)\end{array}$ & $P$-value \\
\hline Age, years & $54.3 \pm 15.6$ & $55.2 \pm 14.7$ & $52.8 \pm 17.2$ & 0.55 \\
\hline Sex, Male/Female & $35 / 49$ & $21 / 31$ & $14 / 18$ & \\
\hline \multicolumn{5}{|c|}{ Pemphigus subtype, $n(\%)$} \\
\hline Vulgaris & $53(63.1)$ & $32(61.5)$ & $21(65.6)$ & \\
\hline Foliaceus & $28(33.3)$ & $17(32.7)$ & $11(34.4)$ & \\
\hline Vegetans & $3(3.6)$ & $3(5.8)$ & $0(0)$ & \\
\hline PDAI score & $31.6 \pm 21.7$ & $23.8 \pm 14.3$ & $44.2 \pm 25.6$ & $<0.001$ \\
\hline \multicolumn{5}{|l|}{ Severity, $n(\%)$} \\
\hline Severe & $41(48.8)$ & 19 (36.5) & $22(68.8)$ & 0.007 \\
\hline Moderate & $43(51.2)$ & $33(63.5)$ & $10(31.2)$ & \\
\hline ELISA index & $755.2 \pm 1872.1$ & $755.8 \pm 2137.5$ & $748.1 \pm 1343.1$ & 0.24 \\
\hline
\end{tabular}

ELISA, enzyme-linked immunosorbent assay; PDAI, pemphigus disease area index.

period was $5.0 \pm 2.0$ years (mean $\pm \mathrm{SD}$, range: $2.1-8.7$ years). The study population of 84 patients included 35 males and 49 females with a mean age of $54.3 \pm 15.6$ years (mean \pm SD, range: $16-90$ years) at the start of treatment. The mean PDAI before initiation of therapy was $31.6 \pm 21.7$ (mean $\pm \mathrm{SD}$, range: 9-117), corresponding to 41 cases of severe disease and 43 of moderate disease, according to the severity grading. ${ }^{13}$ The ELISA titre of autoantibodies against Dsg1 or Dsg3 was $755.2 \pm 1872.1$ (mean \pm SD, range: $23.2-14$ 993.9). Previous treatment of patients transferred to our department from other hospitals consisted of systemic CS at a dose of $0.2-0.8 \mathrm{mg} / \mathrm{kg} /$ day (equivalent to PSL) in 11 patients, and of $1 \mathrm{mg} / \mathrm{kg} /$ day in combination with immunosuppressants in five patients, dapsone (diaminodiphenyl sulphone) in one patient, and PE in one patient.

\section{Remission rate in patients treated based on the JG}

Disease control and the end of the consolidation phase, and thus CR on therapy, were achieved successfully in 83 of the 84 patients $(98.8 \%$ ) (Table 2). Only one patient with PV did not achieve CR. The time to remission was $13.9 \pm 9.4$ months (mean \pm SD, range: 6.6-84.6 months). Remission 1 year after initial therapy was noted in 58 patients $(69.0 \%)$, and at 2 years after initial therapy was documented in 78 patients $(92.9 \%)$. In the patient with PV in whom disease remission was not achieved, disease activity was not progressive, instead being mild and stable, based on treatment with $<20 \mathrm{mg}$ PSL/day. Relapse occurred in 12 patients (14.3\%): in six during tapering of the CS dose before remission was reached and in six after achieving remission on therapy. In all 12 patients, remission was treated by increasing the CS dose, or adding or switching immunosuppressive agents and/or anti-CD20 antibody therapy. The initial daily dose of PSL was $1 \mathrm{mg} / \mathrm{kg} /$ day in 77 patients (91.7\%); the remaining seven patients $(8.3 \%)$ were initially treated with $0.5 \mathrm{mg} / \mathrm{kg} /$ day. Eleven patients $(13.1 \%)$ were given PSL alone due to a history of malignancy and their advanced age. In the remaining 73 patients (86.9\%), immunosuppressive agents were added to the CS regimen: AZP in 69 patients and cyclosporine in four patients. Other additional therapies were needed in 32 patients $(38.1 \%)$, including IVIG in 23, PE in 20, steroid pulse therapy in 17 and intravenous cyclophosphamide in 3 (and where in most cases these additional therapies were administered in various combinations). In the nine patients resistant to these treatments, a human anti-CD20 monoclonal antibody (either rituximab, $n=8$; or ofatumumab, $n=1$ ) was administered. Between the patients requiring additional therapy [additional therapy $(+)]$ and those who did not require additional therapy [additional therapy $(-)$ ], we found significant differences in PDAI score $(23.8 \pm 14.3$ vs. $44.2 \pm 25.6$, respectively, $P<0.001$; Fig. 1a), severity category before initial treatment (36.5\% vs. $68.8 \%$, respectively, in 'Severe' and $63.5 \%$ vs. $31.2 \%$, respectively, in 'Moderate', $P=0.007$ ) (Table 1), cumulative proportion of remission as determined by Kaplan-Meier estimates (Fig. 2, $P=0.003$ ) and remission rates within 2 years after initiation of treatment $(98.1 \%$ vs. $84.4 \%$, respectively, $P=0.03$; Table 2). The relationship between PDAI score before initial treatment and the time to remission was assessed (Fig. 3). Remission mostly occurred within 7-24 months regardless of disease activity. An approximation straight line indicated no obvious relationship between PDAI score and time to remission $\left(R^{2}=0.01\right)$. Comparison of PDAI score before initial treatment between the groups that had and had not achieved remission within 1 year (Fig. 4a) indicated no significant difference $(30.3 \pm 21.8$ vs. $34.5 \pm 21.6$, respectively, $P=0.42)$. Data on the ELISA indices (Dsg1 for PF, Dsg1 or Dsg3 for PV, and Dsg3 for pemphigus vegetans) were available for 79 patients. At the time of remission, the ELISA index values decreased from the original level (average change of $9.9 \%$ ) as follows: $<10 \%$ $(n=53,67.1 \%), 10-20 \%(n=14,17.7 \%), 20-50 \% \quad(n=10$, $12.7 \%)$ and $>50 \%(n=2,2.5 \%$; Table 2$)$. The ELISA index 
Table 2 The results of treatments based on the JG

\begin{tabular}{|c|c|c|c|c|}
\hline & All cases $(n=84)$ & $\begin{array}{l}\text { Additional therapy (-) } \\
(n=52)\end{array}$ & $\begin{array}{l}\text { Additional therapy }(+) \\
(n=32)\end{array}$ & $P$-value \\
\hline Remission, $n(\%)$ & $83(98.8)$ & $52(100)$ & $31(96.9)$ & \\
\hline First year & $58(69.0)$ & $40(76.9)$ & $18(56.3)$ & 0.06 \\
\hline (Severe/Moderate) & $27 / 31$ & $14 / 26$ & $13 / 5$ & \\
\hline Second year & 78 (92.9) & $51(98.1)$ & $27(84.4)$ & 0.03 \\
\hline (Severe/Moderate) & $37 / 41$ & $19 / 32$ & $18 / 9$ & \\
\hline Duration to remission, months & $13.9 \pm 9.4$ & $11.8 \pm 4.2$ & $17.3 \pm 13.9$ & 0.10 \\
\hline Relapse, $n(\%)$ & $12(14.3)$ & $6(11.5)$ & $6(18.8)$ & 0.52 \\
\hline Before remission & $6(7.1)$ & $2(3.8)$ & $4(12.5)$ & \\
\hline After remission & $6(7.1)$ & $4(7.7)$ & $2(6.3)$ & \\
\hline \multicolumn{5}{|l|}{ Initial treatment, $n(\%)$} \\
\hline PSL (1 mg/kg/day) & 77 (91.7) & $46(88.5)$ & $31(96.9)$ & \\
\hline With AZP or CyA/alone & $69 / 8$ & $41 / 5$ & $28 / 3$ & \\
\hline PSL (0.5mg/kg/day) & $7(8.3)$ & $6(11.5)$ & $1(3.1)$ & \\
\hline With AZP or CyA/alone & $4 / 3$ & $3 / 3$ & $1 / 0$ & \\
\hline AZP & $69(82.1)$ & $41(78.8)$ & $28(87.5)$ & \\
\hline СуA & $4(4.8)$ & $3(5.8)$ & $1(3.1)$ & \\
\hline Additional therapy, $n(\%)$ & $32(38.1)$ & & $32(100)$ & \\
\hline IVIG & $23(27.4)$ & & $23(71.9)$ & \\
\hline PE & $20(23.8)$ & & $20(62.5)$ & \\
\hline Steroid pulse & $17(20.2)$ & & $17(53.1)$ & \\
\hline Anti-CD20 antibody, RTx/Oft & $9,8 / 1(10.7)$ & & $9,8 / 1(28.1)$ & \\
\hline IVCY & $3(3.6)$ & & $3(9.4)$ & \\
\hline Changes in the ratio of ELISA indexes, $n(\%)$ & $n=79$ & $n=50$ & $n=29$ & \\
\hline$<10 \%$ & $53(67.1)$ & $33(66.0)$ & $20(69.0)$ & \\
\hline $10-20 \%$ & $14(17.7)$ & $8(16.0)$ & $6(20.7)$ & \\
\hline $20-50 \%$ & $10(12.7)$ & $8(16.0)$ & $2(6.9)$ & \\
\hline$>50 \%$ & $2(2.5)$ & $1(2.0)$ & $1(3.4)$ & \\
\hline Adverse effects & $67(79.8)$ & $38(73.1)$ & $29(90.6)$ & 0.09 \\
\hline Opportunistic or severe infections & $9(10.7)$ & $1(1.9)$ & $8(25.0)$ & 0.002 \\
\hline$\geq$ Grade 3 & $38(45.2)$ & $16(30.8)$ & $22(68.8)$ & 0.001 \\
\hline
\end{tabular}

AZP, azathioprine; CyA, cyclosporine; ELISA, enzyme-linked immunosorbent assay; IVCY, intravenous cyclophosphamide; IVIG, high-dose intravenous immunoglobulin; JG, Japanese guidelines for the management of pemphigus; Oft, ofatumumab; PE, plasma exchange; PSL, prednisone; Rtx, rituximab.

values before treatment did not differ significantly between the additional therapy (-) and additional therapy (+) groups $(755.8 \pm 2137.5$ vs. $748.1 \pm 1343.1$, respectively, $P=0.24)$

(Fig. 1b), or between the groups of patients who had and had not achieved remission within 1 year $(732.8 \pm 2004.8$ vs. $774.2 \pm 1507.8$, respectively, $P=0.92$; Fig. $4 \mathrm{~b})$.

\section{Safety}

Adverse events (AEs) were defined and categorized according to the Common Terminology Criteria for adverse events (version 5.0) that was issued by the cancer therapy evaluation programme in National cancer institute. In this study, AEs developed in 67 patients (79.7\%, Table 3). A 90-year-old patient died after gastrointestinal haemorrhage during the observation period, but it was unclear if this was directly attributable to adverse effects caused by treatment. The AEs consisted of infection $(n=56)$, serum liver enzyme elevation $(n=38)$, diabetes $(n=19)$, hyperlipidaemia $(n=9)$, hypertension, decreased white blood cell count and drug eruption $(n=7)$, decreased platelet count and osteoporosis $(n=5)$ and spinal fracture $(n=4)$. AEs $\geq$ grade 3 occurred in 38 patients (45.2\%). All infections (57 infectious adverse events occurred in 37 patients) were treated and cured with the appropriate antibiotic, antiviral and antifungal drugs, although opportunistic and severe infections (CMV hepatitis, $n=6$; bacteraemia, $n=2$; pneumocystis carinii pneumonia, nocardia pneumonia, cryptococcus pneumonia and reactivation of HBV, $n=1$ each) were observed in nine patients who had been treated with $1 \mathrm{mg} / \mathrm{kg} /$ day of PSL and more than one adjuvant therapy due to their disease activity. All of these patients had multiple other side-effects. The average age distribution of these nine patients was slightly higher than that of those who did not have severe infections $(65.4 \pm 12.7$ vs. $52.9 \pm 6.6$, respectively, $P=0.07)$. 
(a)

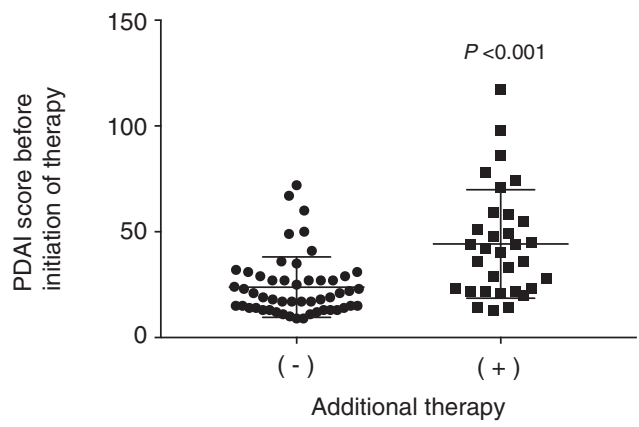

(b)

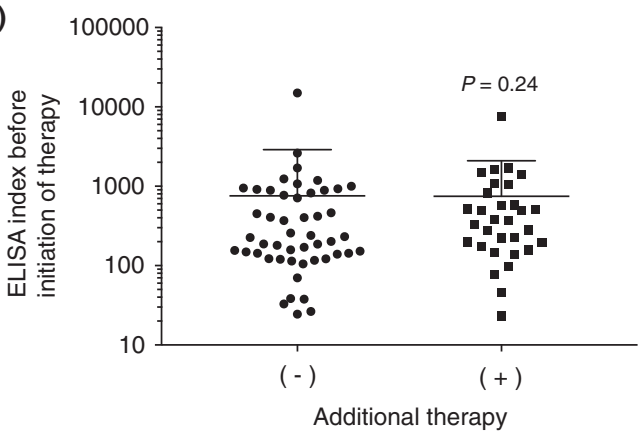

Figure 1 A comparison of PDAl score (a) and ELISA index (b) before initiation of therapy between the groups that did and did not require additional therapy.

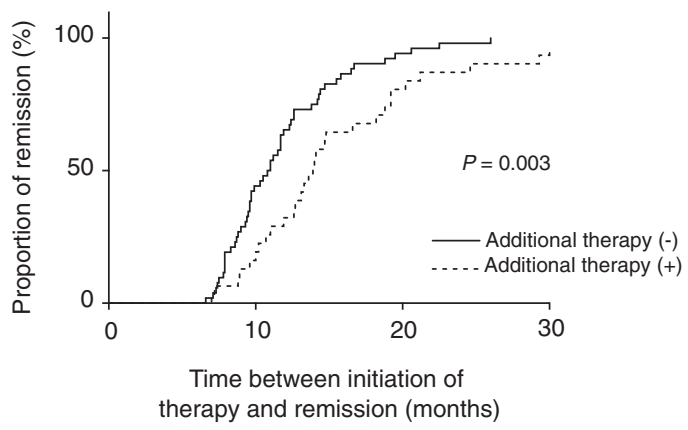

Figure 2 Cumulative proportion of remission on therapy in the groups that did and did not require additional therapy (KaplanMeir's methods).

A case with a decreased number of white blood cells and the other case with platelet decrease of grade 4 were treated with granulocyte colony stimulating factor and platelet transfusion, respectively. Serum liver enzyme elevation, including grade 4, was reversed by dose reduction or cessation of either AZP or trimethoprim-sulfamethoxazole, which was used to prevent pneumocystis carinii pneumonia. Other AEs, particularly diabetes, were also treated or improved gradually during tapering of the CS dose. There were significant differences between the

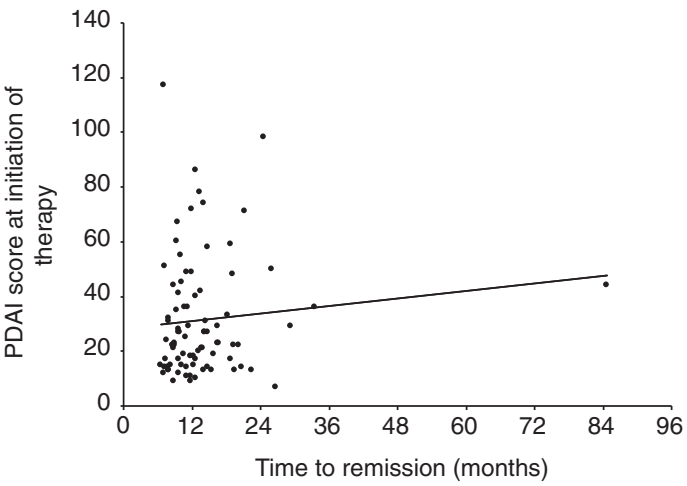

Figure 3 Relations between PDAI score at initiation of therapy and the time to remission with an approximation straight line $\left(y=0.2339 x+28.13, R^{2}=0.01\right)$.

(a)

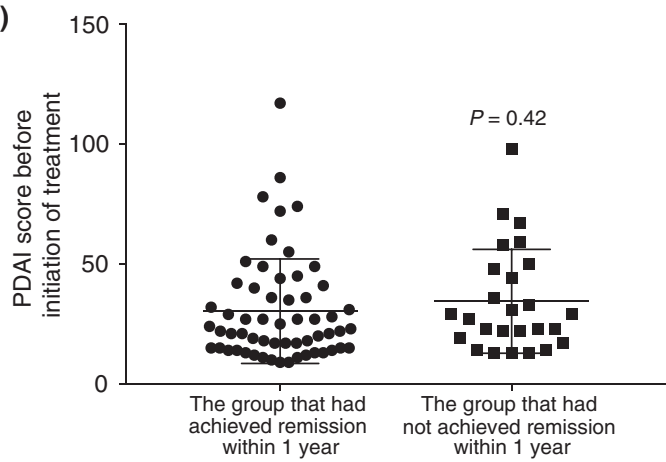

(b)

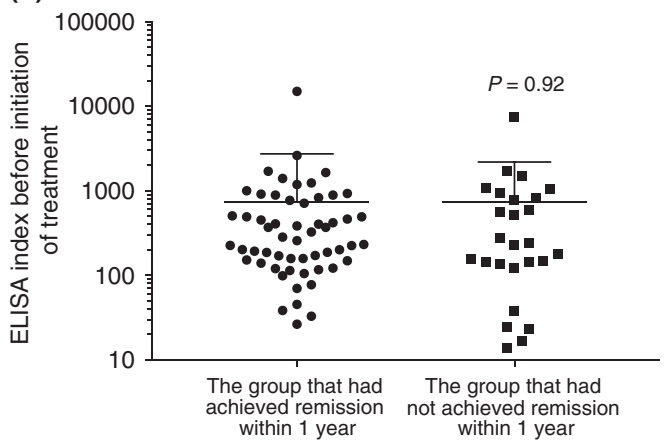

Figure 4 A comparison of PDAl score (a) and ELISA index (b) before initiation of treatment between the groups that had and had not achieved remission within 1 year.

additional therapy $(-)$ and additional therapy $(+)$ groups in the occurrence of severe infections $(1.9 \%$ vs. $25.0 \%$, respectively, $P=0.002)$ and AEs $\geq$ grade $3(30.8 \%$ vs. $68.8 \%$, respectively, $P=0.001)$, while the number of cases with overall AEs was not significantly different between the two groups (73.1\% vs. $90.6 \%$, respectively, $P=0.09$; Table 2 ). 
Table 3 Adverse effects recorded during the follow-up period

\begin{tabular}{|c|c|c|}
\hline \multirow[t]{2}{*}{ Adverse effects $(n=67)$} & \multicolumn{2}{|l|}{ Cases } \\
\hline & Any grade & Grade $3 / 4$ \\
\hline Liver enzyme elevation & 38 & $16 / 3$ \\
\hline Infection & 37 & \\
\hline Skin infection & 18 & $9 / 0$ \\
\hline Mucosal infection & 13 & \\
\hline Viral hepatitis & 7 & $7 / 0$ \\
\hline Lung infection & 7 & $3 / 0$ \\
\hline Pustular rash & 5 & \\
\hline Bacteraemia & 2 & $2 / 0$ \\
\hline Eye infection & 1 & \\
\hline Otitis media & 1 & \\
\hline Paronychia & 1 & \\
\hline Soft tissue infection & 1 & \\
\hline Infective conjunctivitis & 1 & \\
\hline Diabetes & 19 & $12 / 0$ \\
\hline Hyperlipidaemia & 9 & $1 / 0$ \\
\hline Hypertension & 7 & $1 / 0$ \\
\hline White blood cell (including neutrophil) decrease & 7 & $1 / 2$ \\
\hline Drug eruption (maculopapular rash) & 7 & \\
\hline Platelet decrease & 5 & $1 / 1$ \\
\hline Osteoporosis & 5 & \\
\hline Spinal fracture & 4 & \\
\hline Cataract & 2 & \\
\hline Creatinine increase & 2 & \\
\hline Dysgeusia & 2 & \\
\hline Others $\uparrow$ & 19 & $6 / 0$ \\
\hline Death & 1 & \\
\hline
\end{tabular}

$\dagger$ One patient each (Grade 3/4) with vomiting (1/0), thromboembolic event $(1 / 0)$, peripheral motor neuropathy $(1 / 0)$, treatment related secondary malignancy $(1 / 0)$, hypogammaglobulinaemia (1/0), irregular menstruation (1/0), chronic kidney disease, malaise, supraventricular tachycardia, dry eye, multiple posterior pole retinal pigment epithelial disease, central serous retinal chorioretinopathy, insomnia, psychosis, gingival hyperplasia, hyperuricaemia, avascular necrosis, anaemia, alopecia.

\section{Discussion}

We validated the treatment strategy for pemphigus according to the JG. After the first and second year of initial treatment, $69.0 \%$ and $92.9 \%$ of patients achieved remission, respectively, clearly suggesting the efficacy of these guidelines. While our results cannot be easily compared with those of previous studies, given the scarcity of comparable studies and differences in the assessed parameters (e.g. end point), a high rate of remission was also reported in a previous study. ${ }^{14}$ The standard initial dose of PSL was $1 \mathrm{mg} / \mathrm{kg} / \mathrm{day}$ in both this previous study and our study. The JG emphasizes the importance of initial therapy to reach CR without relapse during reduction of CS dosage. ${ }^{4}$ In this study, 12 patients (14.3\%) experienced disease relapse during the course of treatment, which was lower than the rate of relapse in previous studies (20.1-81.8\%). ${ }^{14-20}$ The reasons for the high remission rate and low relapse rate among our patients are still unclear, but in addition to the goal of this study being remission on therapy, the combined use of adjuvant therapy with CS, including immunosuppressive agents, from the beginning of treatment may have affected the outcome, as suggested in a previous report. ${ }^{21}$ Because anti-CD20 antibody therapy was not yet approved for pemphigus in Japan, a few cases treated with rituximab and ofatumumab under clinical trials were included in this study. Recent study showed that administrating rituximab as a first-line therapy could produce high remission rate in pemphigus. ${ }^{22}$ In the near future, introduction of rituximab in pemphigus will strongly affect the guideline and results of pemphigus treatment.

To investigate refractory cases, the study population was divided into two groups that did and did not require additional therapy, and some factors before treatment and the results of treatment were compared between these groups. We found that the additional therapy $(+)$ group had higher PDAI scores, severity before treatment and lower cumulative proportion of remission; moreover, more patients in this group suffered from severe adverse effects, including severe infections. On the other hand, there were no significant differences in age or ELISA index before treatment, time until remission or the relapse and overall adverse effects rate between the groups. These observations suggested that recalcitrant cases may potentially have higher disease activity and severe adverse effects, as well as a lower remission rate within 2 years after treatment. It is important to monitor adverse events carefully when starting additional therapy in refractory cases.

The interval from initiation of therapy to remission for most patients in this study was between 7 and 24 months, and was not correlated with disease severity at treatment initiation as evaluated by the clinical symptom score (PDAI) and serum autoantibody titre (ELISA index). These results highlight the difficulty of predicting the clinical course before treatment. In fact, some patients with severe disease responded well to treatment within a short period, whereas others showed disease of relatively low severity that was refractory to therapy. Therefore, all patients should be monitored carefully and the clinical course in all cases should be assessed during the consolidation phase through initial therapies. The prolonged administration of CS and additional therapies, particularly in patients with refractory disease, can increase the risk of side-effects.

In this study, AEs occurred in 67 patients (79.7\%), including 38 (45.2\%) with AEs $\geq$ grade 3 . This rate was higher than in previous studies $\left(53.7-81.8 \%^{16,23,24}\right.$ ), highlighting that treatments for pemphigus could be life-threatening. Infections accounted for the majority of adverse events, as reported previously. ${ }^{16,24}$ Our results suggested a higher risk of opportunistic and severe infections in patients with multiple additional therapies, along with a variety of other AEs including severe white blood cell decrease, multiple infections and diabetes. Moreover, these patients were older than those who did not have severe infections. Although the mortality rate was lower $(1.2 \%)$ in our cohort than in previous studies $\left(19.7 \%{ }^{25}\right.$ and $\left.12 \%{ }^{26}\right)$, it is 
difficult to compare the data because of differences in patient populations and statistical methods among the studies.

This study has limitation as a retrospective study. The results of the present study suggested the advantages of following the JG for treatment of pemphigus, based on the high remission rate and low rates of relapse. However, it is necessary to bear in mind that AEs occurred at a rate of about $80 \%$ and careful monitoring and appropriate measurements are required. Prospective studies with larger cohorts and longer observation periods are needed to validate our results. Nonetheless, our findings will contribute to the optimization of treatment strategies for patients with pemphigus, such that the therapeutic effects are maximized (as long as adverse effects are managed well).

\section{References}

1 Bystryn JC. Adjuvant therapy of pemphigus. Arch Dermatol 1984; 120: 941-951.

2 Harman KE, Albert S, Black MM. Guidelines for the management of pemphigus vulgaris. Br J Dermatol 2003; 149: 926-937.

3 Harman KE, Brown D, Exton LS et al. British Association of Dermatologists' guidelines for the management of pemphigus vulgaris 2017. Br J Dermatol 2017; 177: 1170-1201.

4 Committee for Guidelines for the Management of Pemphigus Disease, Amagai M, Tanikawa A et al. Japanese guidelines for the management of pemphigus. J Dermatol 2014; 41: 471-486.

5 Ruocco E, Wolf R, Ruocco V, Brunetti G, Romano F, Lo Schiavo A. Pemphigus: associations and management guidelines: facts and controversies. Clin Dermatol 2013; 31: 382-390.

6 Hertl M, Jedlickova H, Karpati S et al. Pemphigus. S2 Guideline for diagnosis and treatment-guided by the European Dermatology Forum (EDF) in cooperation with the European Academy of Dermatology and Venereology (EADV). J Eur Acad Dermatol Venereol 2015; 29: 405-414.

7 Eming R, Sticherling M, Hofmann SC et al. S2k guidelines for the treatment of pemphigus vulgaris/foliaceus and bullous pemphigoid. J Dtsch Dermatol Ges 2015; 13: 833-844.

8 Joly P, Bernard P, Bedane C, Prost C, Ingen-Housz-Oro S. Pemphigus. Guidelines for the diagnosis and treatment. Centres de référence des maladies bulleuses auto-immunes. Société Française de Dermatologie. Ann Dermatol Venereol 2011; 138: 252-258.

9 Feliciani C, Cozzani E, Marzano AV et al. Italian guidelines in pemphigus - adapted from the European Dermatology Forum (EDF) and European Academy of Dermatology and Venereology (EADV). G Ital Dermatol Venereol 2018; 153: 599-608.
10 Murrell DF, Dick S, Ahmed AR et al. Consensus statement on definitions of disease, end points, and therapeutic response for pemphigus. J Am Acad Dermatol 2008; 58: 1043-1046.

11 Rosenbach M, Murrell DF, Bystryn JC et al. Reliability and convergent validity of two outcome instruments for pemphigus. J Invest Dermatol 2009; 129: 2404-2410.

12 Rahbar Z, Daneshpazhooh M, Mirshamas-Shahshahani M et al. Pemphigus disease activity measurements: pemphigus disease area index, autoimmune bullous skin disorder intensity score, and pemphigus vulgaris activity score. JAMA Dermatol 2014; 150: 266-272.

13 Shimizu T, Takebayashi T, Sato Y et al. Grading criteria for disease severity by pemphigus disease area index. J Dermatol 2014; 41: 969-973.

14 Khaled A, Taazayet SB, Ben Alaya N et al. The course and prognosis of pemphigus in 47 Tunisian patients. J Eur Acad Dermatol Venereol 2013; 27: 81-85.

15 Kavusi S, Daneshpazhooh M, Farahani F, Abedini R, Lajevardi V, ChamsDavatchi C. Outcome of pemphigus vulgaris. J Eur Acad Dermatol Venereol 2008; 22: 580-584.

16 Svecova D. Pemphigus vulgaris: a clinical study of 44 cases over a 20 -year period. Int J Dermatol 2015; 54: 1138-1144.

17 Mimouni D, Bar H, Gdalevich M, Katzenelson V, David M. Pemphigus, analysis of 155 patients. J Eur Acad Dermatol Venereol 2010; 24: 947-952.

18 Zaraa I, Mokni M, Hsairi M et al. Pemphigus vulgaris and pemphigus foliaceus: similar prognosis? Int J Dermatol 2007; 46: 923-926.

19 Esmaily N, Ehsani A, Noormohammadpour P, Baiagouy I, Sayanjali S. Pemphigus vulgaris recurrence rate and its relationship with initial corticosteroid dose. Iran J Dermatol 2010; 13: 118-121.

20 Abdolsamadi HR, Abdollahzadeh S, BakianianVaziri P, Beheshti A, Shafigh E, Vehedi M. Epidemiology of pemphigus in Tehran, Iran: a 20-year retrospective study. J Dent Res Dent Clin Dent Prospects 2007; 1: 108-113.

21 Atzmony L, Hodak E, Leshem YA et al. The role of adjuvant therapy in pemphigus: a systematic review and meta-analysis. J Am Acad Dermatol 2015; 73: 264-271.

22 Joly P, Maho-Vaillant M, Prost-Squarcioni C et al. First-line rituximab combined with short-term prednisone versus prednisone alone for the treatment of pemphigus (Ritux 3): a prospective, multicenter, parallelgroup, open-label randomized trial. Lancet 2017; 389: 2031-2040.

23 Alsaleh QA, Nanda A, Al-Baghli NM, Dvorak R. Pemphigus in Kuwait. Int J Dermatol 1999; 38: 351-356.

24 Chams-Davatchi C, Valikhani M, Daneshpazhooh M et al. Pemphigus: analysis of 1209 cases. Int J Dermatol 2005; 44: 470-476.

25 Kridin K, Sagi SZ, Bergman R. Mortality and cause of death in patients with pemphigus. Acta Derm Venereol 2017; 97: 607-611.

26 Langan SM, Smeeth L, Hubbard R, Fleming KM, Smith CJ, West J. Bullous pemphigoid and pemphigus vulgaris-incidence and mortality in the UK: population based cohort study. BMJ 2008; 337: a180. 